\title{
Caracterização térmica e morfológica de fibras contínuas de basalto
}

\section{(Thermal and morphological characterization of basalt continuous fibers)}

\author{
M. A. Schiavon 1 , S. U. A. Redondo', I. V. P. Yoshida \\ ${ }^{1}$ Departamento de Ciências Naturais, Universidade Federal de S. João Del Rei - UFSJ, Campus Dom Bosco, S. J. \\ Del Rei, MG 36301-160 \\ ${ }^{2}$ Instituto de Química, Universidade Estadual de Campinas - UNICAMP, C.P. 6154, Campinas, SP 13083-970 \\ schiavon@ufsj.edu.br
}

\begin{abstract}
Resumo
Os basaltos são rochas vulcânicas abundantes provenientes de extensos derrames de lava. A tecnologia de fiação contínua produz filamentos de basalto na forma de fios micrométricos amorfos. Neste trabalho são apresentados resultados da caracterização térmica e morfológica de fibras longas de basalto, de diâmetro micrométrico. Estas fibras apresentaram excelente estabilidade térmica, resultando em alterações de massa menor que $1 \%$ sob aquecimento até a temperatura de $1000{ }^{\circ} \mathrm{C}$, em atmosfera inerte ou oxidante. Por outro lado, a $\sim 800{ }^{\circ} \mathrm{C}$ ocorreu a cristalização parcial das fibras de basalto, com a formação de fases cristalinas de silicatos e alumino-silicatos, como a anortita, da classe dos plagioclásios. A morfologia das fibras, aquecidas a $1000^{\circ} \mathrm{C}$, foi alterada devido à fusão e cristalização das fases presentes.
\end{abstract}

Palavras-chave: cerâmica, fibras de basalto.

\begin{abstract}
The basalts are abundant volcanic rocks formed from extensive wastes. The technology of continuous wiring produces basalt filaments in micrometric amorphous long fibers. In this work are presented results of the thermal and morphologic characterization of micrometric basalt fibers. These fibers showed excellent thermal stability, resulting in low weight change (less than 1 wt.\%) up to $1000^{\circ} \mathrm{C}$, under heating in inert or oxidant atmosphere. On the other hand, partial crystallization of basalt fibers occurred at $\sim$ $800^{\circ} \mathrm{C}$, resulting in diffraction peaks in the X-ray patterns, characteristic of silicates and aluminosilicates as the anorthite from the plagioclase class. The morphology of fibers after heating at $1000^{\circ} \mathrm{C}$ was changed due to the fusion and crystallization of the present phases.
\end{abstract}

Keywords: ceramic, basalt fiber.

\section{INTRODUÇÃO}

Os basaltos são rochas vulcânicas abundantes no planeta e formam derrames extensos de lava em muitas regiões. Pertencem à categoria das rochas ígneas, as quais são formadas pelo resfriamento e conseqüente solidificação do magma, o qual é constituído principalmente por óxidos de silício, alumínio, ferro, cálcio, magnésio, sódio e potássio. O basalto é constituído basicamente por minerais de silício, alumínio e ferro, na forma de piroxênios (augita), plagioclásios (labradorita) e magnetita [1]. A composição química aproximada do basalto é de 43 a $47 \%$ de $\mathrm{SiO}_{2}$, 11 a $13 \%$ de $\mathrm{Al}_{2} \mathrm{O}_{3}, 10$ a $12 \%$ de $\mathrm{CaO}$ e 8 a $10 \%$ de $\mathrm{MgO}$, além de outros óxidos que estão presentes em percentagens inferiores a $5 \%[2,3]$.

O basalto é muito abundante na Rússia e a simplicidade da tecnologia de processamento deste material torna seus produtos relativamente baratos. É empregado principalmente na forma de produtos moldados como lajes, tubos, entre outros, em aplicações que requerem alta resistência à abrasão, a altas temperaturas e ambientes químicos drásticos. Na forma de fibras o basalto ainda é muito pouco empregado, tendo potencialidade de substituir o amianto em muitas aplicações. A tecnologia de fiação contínua, patenteada por russos, pode produzir filamentos de basalto na forma de fios amorfos de diâmetro micrométrico [3]. Quando a rocha basáltica é fundida em temperaturas na faixa de 1500 a $1700{ }^{\circ} \mathrm{C}$, e resfriada rapidamente, forma-se um sólido praticamente amorfo. Se o resfriamento for lento, ocorre cristalização quase completa, formando minerais, principalmente plagioceno e piroxeno, os quais perfazem 80 $\%$ dos muitos tipos de basalto existentes no planeta [3]. No Brasil, há apenas uma patente sobre o processo de produção de fibras de basalto, depositada pela Saint Gobain [4].

$O$ interesse na utilização de fibras contínuas de basalto para o reforço de compósitos tem aumentado significativamente nos últimos anos, devido, principalmente ao crescimento das aplicações de compósitos de matrizes cerâmicas ou poliméricas [5]. Fios micrométricos de basalto podem ser usados para a produção de estruturas 2D ou 3D em compósitos, tecidos de malhas especiais e também linhas para costura, as quais podem ser usadas para unir 
sacos para filtração à quente ou em ambientes quimicamente agressivos, entre outros [3]. A principal vantagem dos materiais produzidos à base de basalto está nas propriedades termofísicas, mecânicas e na alta resistência à corrosão. A resistência ao calor de fibras de basalto $\left(-200\right.$ a $\left.700 / 900{ }^{\circ} \mathrm{C}\right)$ supera a de fibras de vidro $\left(-60\right.$ a $\left.450{ }^{\circ} \mathrm{C}\right)$ e mineral (- 50 a $600{ }^{\circ} \mathrm{C}$ ) [6], e ainda compete, ao menos do ponto de vista econômico, com as fibras de carbono. Além disso, são livres de efeitos carcinogênicos, não poluem o meio ambiente e possuem alta resistência química, pois são mais estáveis que as de vidro na presença de álcalis fortes.

Toropina et al [7] descreveram a obtenção de malhas produzidas com fibras de basalto para serem empregadas em construções, em materiais compósitos, como isolante térmico, entre outras aplicações. Estas malhas apresentaram desempenho satisfatório, sendo recomendada para a substituição de malhas produzidas a partir de fibras de vidro. Já foi também demonstrada a possibilidade da utilização de malhas de fibras de basalto, e fibras de basalto/fibras de vidro, para isolamento elétrico e em construção [8].

$\mathrm{Na}$ área de materiais compósitos estruturais, as fibras de vidro e de carbono são as mais amplamente empregadas [5]. Entretanto, devido às vantagens das fibras de basalto em relação às de vidro e de carbono, como baixo preço e excelentes propriedades mecânicas, estudos envolvendo a utilização de fibras de basalto como cargas de reforço em matrizes variadas como polímeros, cerâmicas e cimentos têm despertado interesses [9-14]. A área de compósitos poliméricos reforçados com fibras é, até o momento, a mais promissora para a utilização destes reforços, pois vantagens adicionais são oferecidas pelas fibras como a baixa higroscopicidade, com teor de umidade menor que $1 \%$, enquanto as fibras de vidro chegam a $20 \%$ [10]. Esta característica é de interesse para a maioria dos sistemas poliméricos [15].

$\mathrm{Na}$ utilização de fibras contínuas de basalto como reforço de compósitos é de interesse o conhecimento das propriedades específicas e da natureza mineral destas fibras. Neste trabalho, são apresentados os resultados obtidos na análise do comportamento térmico, avaliado por termogravimetria (ATG) e análise térmica diferencial (ATD), e da morfologia, avaliada por difração de raios $\mathrm{X}$ (DRX) e microscopia eletrônica de varredura (MEV), de fibras contínuas de basalto.

\section{MATERIAIS E MÉTODOS}

Neste trabalho foram utilizadas fibras longas de basalto, de procedência Albarrie Canada Ltd. (Ontário, Canadá), e com diâmetro aproximado de $9 \mu \mathrm{m}$.

A avaliação térmica das fibras de basalto foi realizada usando simultaneamente termogravimetria e análise térmica diferencial em uma termobalança TA Instruments SDTQ600. As análises foram realizadas sob atmosferas inerte e oxidante, sob fluxo constante de $\mathrm{Ar}$ ou ar sintético de 100 $\mathrm{mL} / \mathrm{min}$, respectivamente, com velocidade de aquecimento de $10{ }^{\circ} \mathrm{C} / \mathrm{min}$, da temperatura ambiente até $1000^{\circ} \mathrm{C}$.
O acompanhamento da cristalização da amostra foi efetuado por difração de raios $\mathrm{X}$ em um difratômetro Shimadzu XRD6000 com radiação $\mathrm{CuK}_{\alpha}(1,5418 \AA$ ), com uma tensão de $30 \mathrm{kV}$ e corrente de $20 \mathrm{~mA}$. O equipamento possui um forno acoplado ao porta-amostra que permite $\mathrm{o}$ aquecimento sob atmosfera controlada. Neste experimento, utilizou-se atmosfera de argônio, e taxa de aquecimento de $10^{\circ} \mathrm{C} / \mathrm{min}$. Difratogramas foram registrados em intervalos de temperatura de $100^{\circ} \mathrm{C}$. Durante o registro do difratograma, a temperatura foi mantida constante. A determinação das fases cristalinas foi efetuada utilizando-se a biblioteca de padrões de difração disponível no equipamento.

A análise morfológica das fibras foi realizada por microscopia eletrônica de varredura em um microscópio JEOL-JSM 6360LV operando com uma voltagem de aceleração de $20 \mathrm{kV}$. As amostras foram acondicionadas em porta-amostras de grafite, e recobertas com uma fina camada de carbono, usando um metalizador modular de alto vácuo BAL-TEC MED 020. Análises elementares foram feitas no mesmo microscópio, acoplado ao analisador de espectroscopia de energia dispersiva (EDS) NORAN SYSTEM SIX, com a mesma voltagem de aceleração usada nas micrografias.

\section{RESULTADOS E DISCUSSÃO}

A fibra de basalto foi inicialmente caracterizada por ATG e ATD. A Fig. 1 ilustra as curvas obtidas em análises simultâneas, sob atmosfera inerte (argônio) e atmosfera oxidante (ar sintético). As curvas de perda de massa em função da temperatura evidenciaram uma alta estabilidade térmica, em ambas as atmosferas, com perdas de massa dentro do erro experimental da medida que é de $1 \%$. As curvas de ATD também apresentaram o mesmo perfil, sendo caracterizadas por um longo e contínuo processo exotérmico, sugerindo um processo contínuo de cristalização. Picos exotérmicos bem definidos apareceram em 807 e $823{ }^{\circ} \mathrm{C}$ nos termogramas obtidos sob atmosferas inerte e oxidante, respectivamente, e

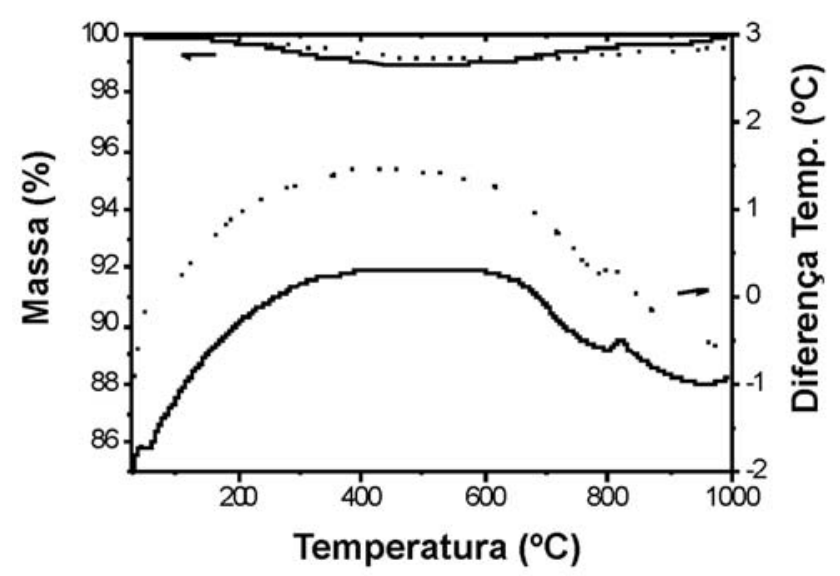

Figura 1: Curvas de ATG e ATD da fibra de basalto. Linhas contínuas: atmosfera oxidante; linhas pontilhadas: atmosfera inerte.

[Figure 1: TGA and DTA curves of the basalt fiber. Solid lines: oxidant atmosphere; dotted lines: inert atmosphere.] 
provavelmente estão relacionados à alta reatividade da fibra amolecida, que se cristaliza.

A análise elementar das fibras de basalto efetuada por EDS, Fig. 2, evidenciou a presença dos seguintes elementos na fibra: $\mathrm{Na}, \mathrm{Mg}, \mathrm{Al}, \mathrm{Si}, \mathrm{P}, \mathrm{K}, \mathrm{Ca}$, Ti e Fe. De fato, o basalto é constituído majoritariamente de silicatos e óxidos de alumínio, além de óxidos de sódio, potássio e fósforo. Fe e Ti são os metais de transição presentes em maior concentração na maioria dos basaltos, seguidos por manganês [2], o qual não foi observado nesta análise. Óxidos metálicos usualmente em composições abaixo de 5\% em massa [3]. O mapeamento por EDS de uma superfície de fratura da fibra (imagem e espectro não apresentados) indicou uma distribuição homogênea destes elementos por toda a fibra.
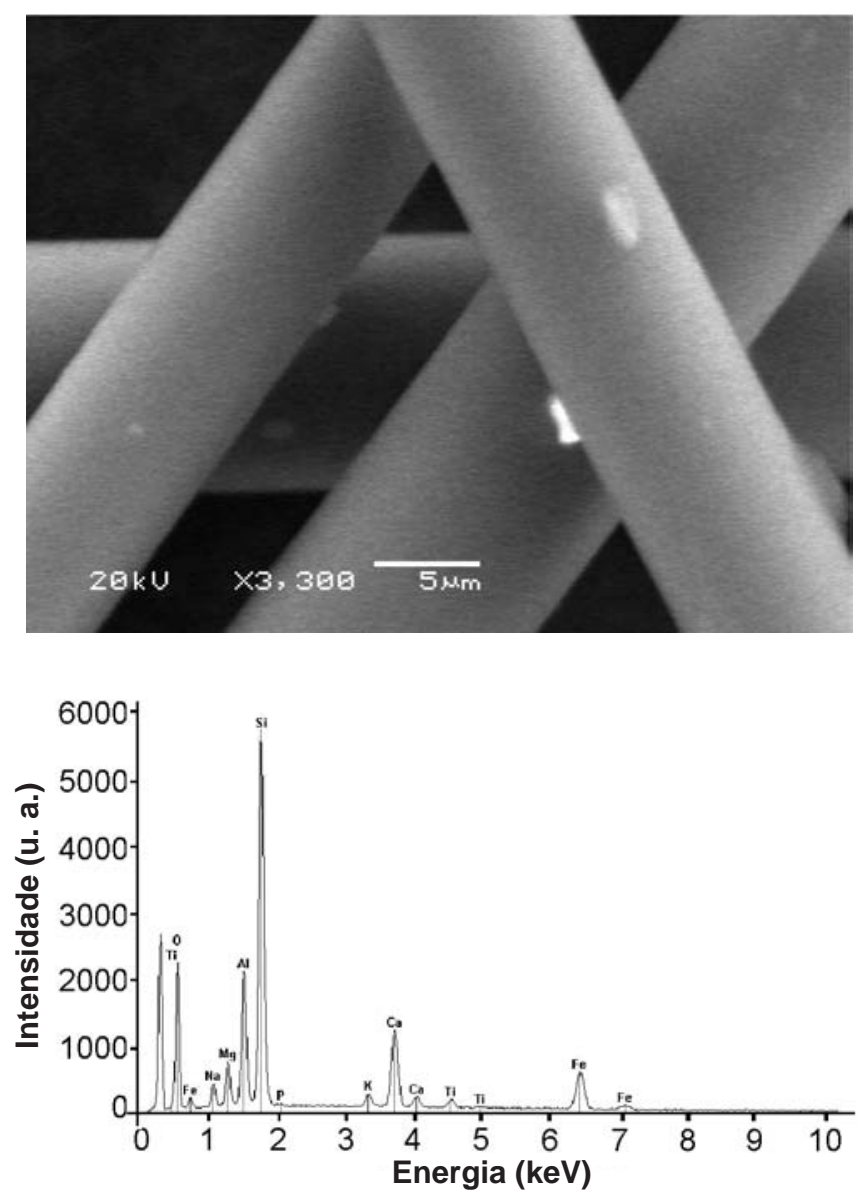

Figura 2: Micrografia de MEV da fibra de basalto com o correspondente espectro de fluorescência (espectroscopia de raios X de energia dispersiva) de toda área.

[Figure 2: SEM micrograph of the basalt fiber with the corresponding fluorescence (energy dispersive X-ray analysis) spectrum of the entire area.]

A Fig. 3 apresenta o estudo da cristalização das fibras de basalto durante aquecimento sob atmosfera inerte de argônio. Os padrões de difração foram registrados in situ no portaamostra aquecido. Assim, as linhas verticais assinaladas no difratograma da Fig. 3 correspondem aos picos de difração do porta-amostra, que é um interferente nesta análise. A análise dos difratogramas evidenciou que as fibras de basalto são predominantemente amorfas até temperaturas da ordem de $700{ }^{\circ} \mathrm{C}$. A temperaturas igual ou superiores a $800{ }^{\circ} \mathrm{C}$ ocorreu a formação preferencial da fase anortita, $\mathrm{Ca}\left[\mathrm{Al}_{2} \mathrm{Si}_{2} \mathrm{O}_{8}\right]$, presente no grupo dos plagioclásios [1]. A $1000{ }^{\circ} \mathrm{C}$, em adição à fase anortita, observou-se a cristalização de silicato de magnésio, $\mathrm{MgSiO}_{3}$ [1], além de outros aluminossilicatos, em menor proporção [1]. Entretanto, mesmo no padrão de difração obtido a $1000{ }^{\circ} \mathrm{C}$, a intensidade dos picos é ainda relativamente baixa evidenciando que nas condições experimentais de análise, a cristalização das fibras é parcial. Uma das limitações da utilização de fibras amorfas de basalto em altas temperaturas é a cristalização que ocorre devido ao amolecimento do material. Observou-se também que a temperaturas superiores a $1000{ }^{\circ} \mathrm{C}$ ocorreu o amolecimento da amostra, levando à perda de forma.

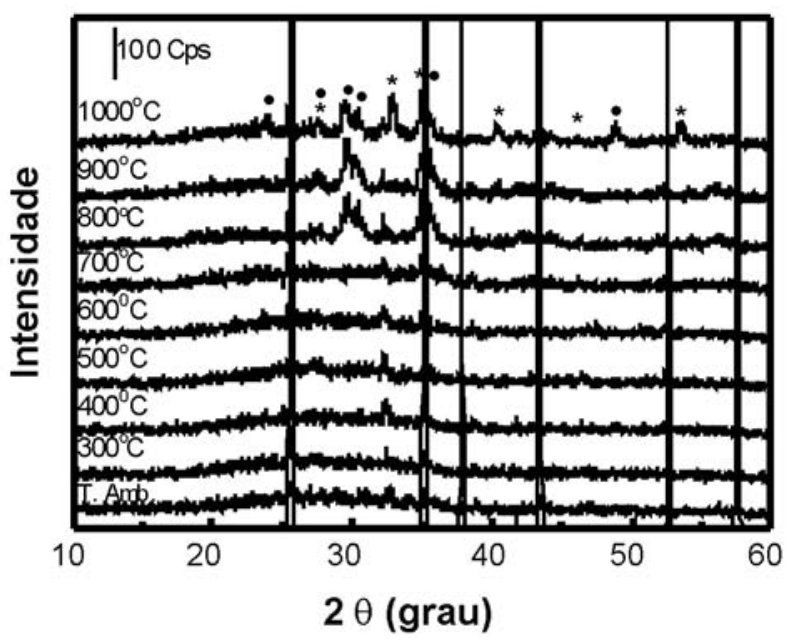

Figura 3: Difratogramas de raios $\mathrm{X}$ das fibras de basalto, registrados durante o aquecimento sob atmosfera de argônio, a $10{ }^{\circ} \mathrm{C} / \mathrm{min}$. Linhas verticais marcadas indicam difração relativas ao portaamostra utilizado. Legenda: ${ }^{*} \mathrm{MgSiO}_{3} ; \bullet \mathrm{CaAl}_{2} \mathrm{Si}_{2} \mathrm{O}_{8}$.

[Figure 3: X-ray diffraction of the basalt fibers, registered during heating under argon atmosphere, at $10^{\circ} \mathrm{C} / \mathrm{min}$. Vertical lines show the diffractions due to the sample holder used. Legend: * $\mathrm{MgSiO}_{3}$; - $\mathrm{CaAl}_{2} \mathrm{Si}_{2} \mathrm{O}_{8}$.]

As micrografias de superfície das fibras de basalto em diferentes amplificações estão apresentadas na Fig. 4. É possível observar que as fibras apresentam superfície lisa, sem detalhes morfológicos nas diferentes ampliações. Além disso, no interior das fibras não há qualquer outro contraste que indique heterogeneidade de composição. Análise de diferentes fibras indicaram espessuras bem definidas e constantes ao longo de toda a extensão, de $\sim 9 \mu \mathrm{m}$.

A temperaturas igual ou superior a $800{ }^{\circ} \mathrm{C}$ observouse a cristalização parcial das fibras, promovida pelo amolecimento do material, resultando em alterações morfológicas. As Figs. 5 e 6 apresentam as micrografias das fibras de basalto, sob diferentes ampliações, aquecidas a 800 

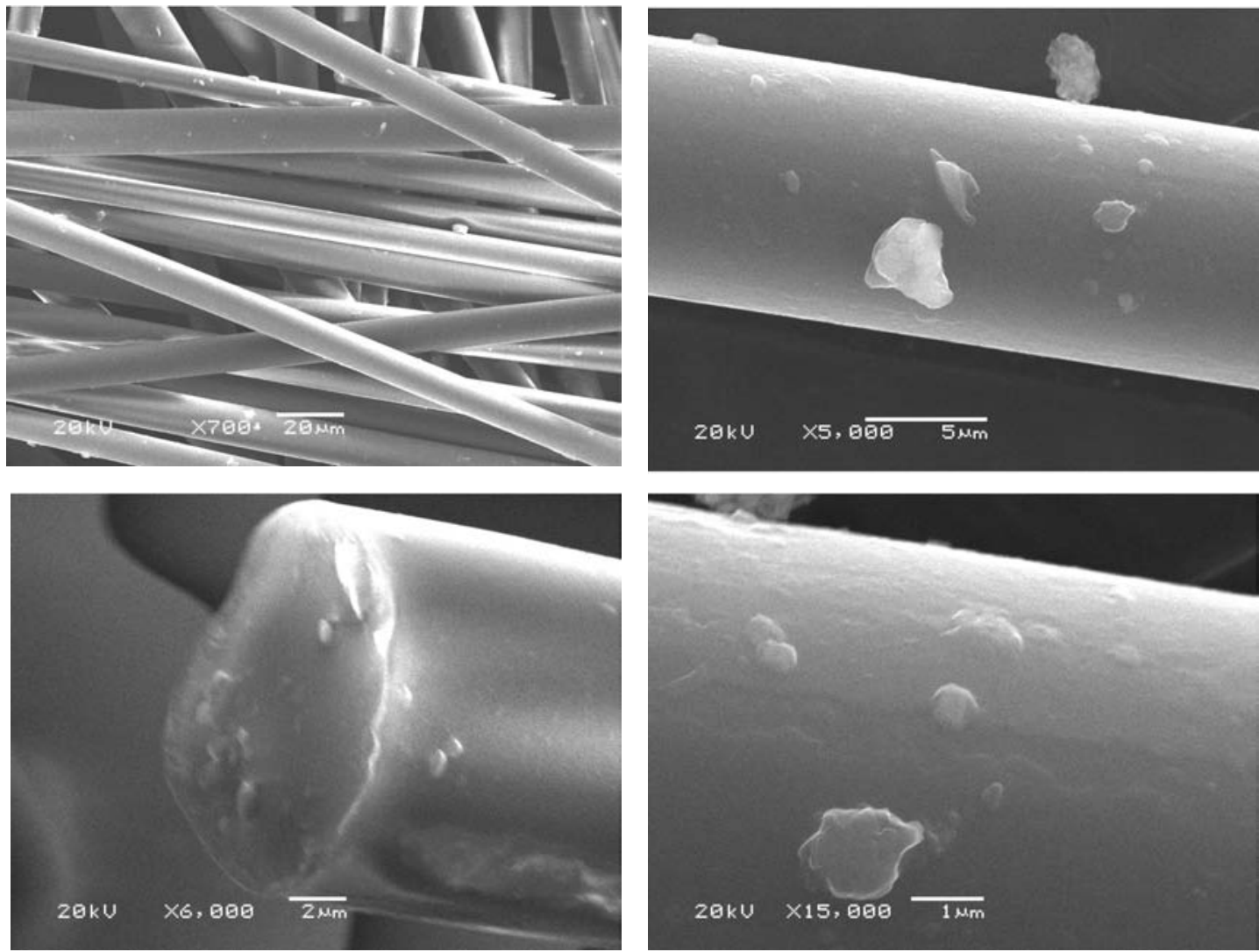

Figura 4: Micrografias MEV da superfície das fibras e da superfície de fratura das fibras de basalto em diferentes ampliações. [Figure 4: SEM micrographs of the fiber surfaces and fracture surface of the basalt fibers in different magnifications.]
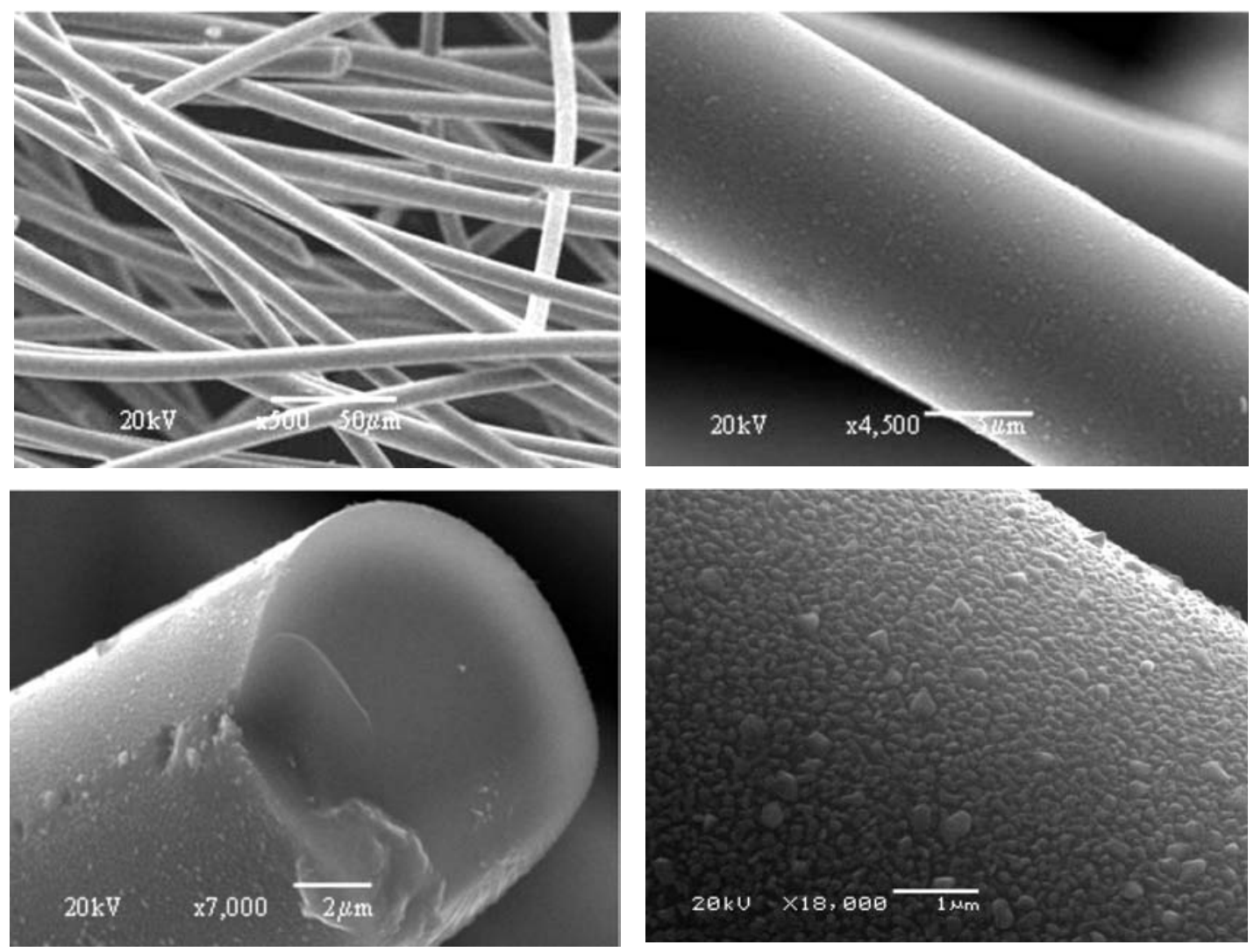

Figura 5: Micrografias MEV da superfície das fibras e da superfície de fratura das fibras de basalto pirolisadas a $800{ }^{\circ} \mathrm{C}$ em diferentes ampliações. [Figure 5: SEM micrographs of the fibers surfaces and fracture surface of the basalt fibers pirolysed at $800^{\circ} \mathrm{C}$ in different magnifications.] 

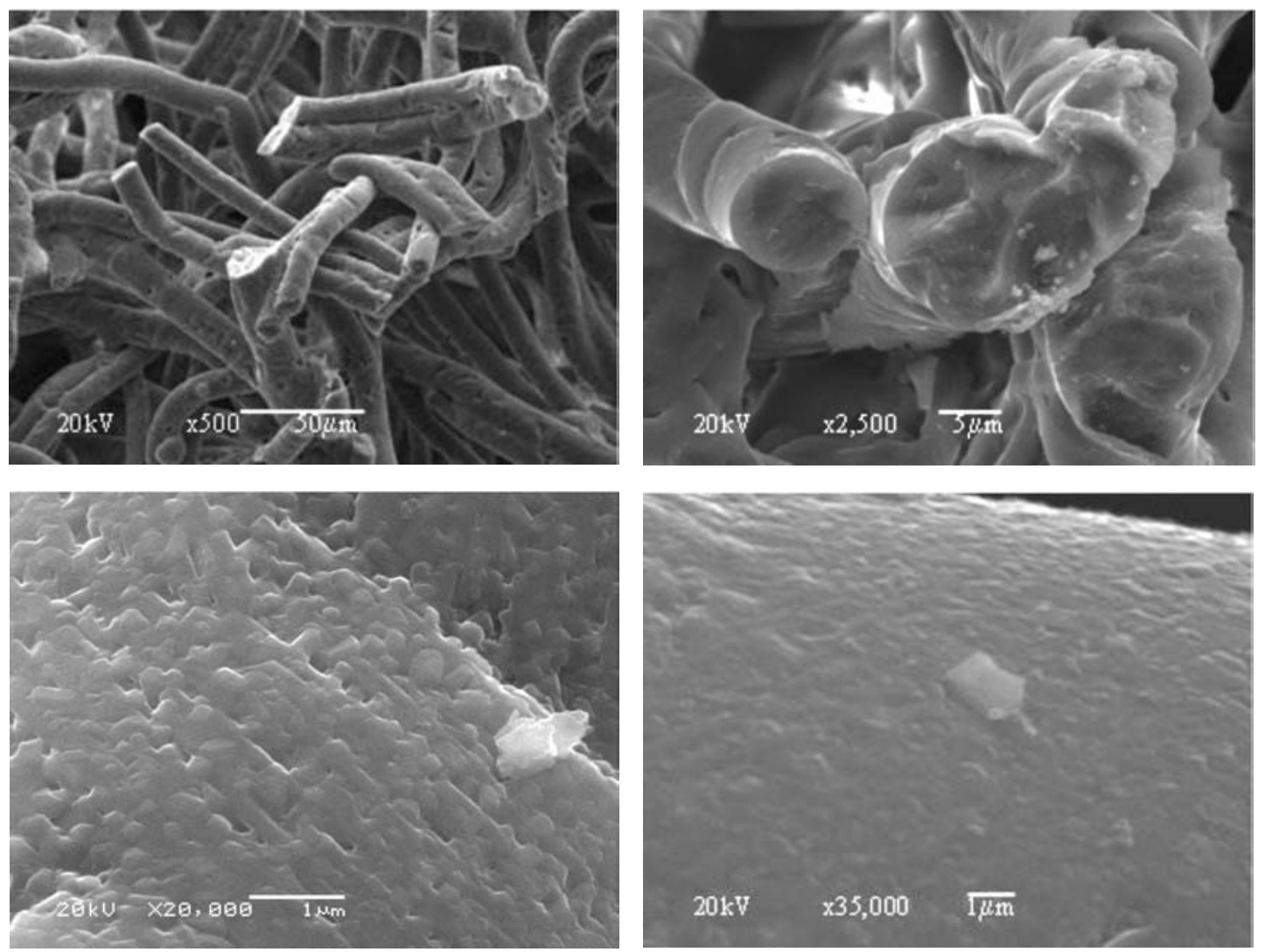

Figura 6: Micrografias MEV da superfície das fibras e da superfície de fratura das fibras de basalto pirolisadas a $1000{ }^{\circ} \mathrm{C}$ em diferentes ampliações.

[Figure 6: SEM micrographs of the fibers surfaces and fracture surface of the basalt fibers pirolysed at $1000{ }^{\circ} \mathrm{C}$ in different magnifications.]

e $1000{ }^{\circ} \mathrm{C}$, respectivamente. Nas fibras aquecidas até $800{ }^{\circ} \mathrm{C}$ pode-se observar a presença de cristalitos, principalmente na superfície das fibras, tornando-as mais rugosas. Em algumas fibras foi possível observar uma ligeira curvatura, provocada pelo seu amolecimento.

As fibras aquecidas a $1000{ }^{\circ} \mathrm{C}$ apresentaram uma superfície rugosa, com morfologia que sugere a aglomeração de cristalitos, provavelmente facilitada pelo maior amolecimento da fase amorfa de basalto. A análise de um conjunto de fibras evidenciou também a aglomeração de algumas fibras promovida pela junção dos contornos amolecidos. Conseqüentemente, nesta temperatura se observaram os maiores danos nas fibras de basalto. A formação dos cristalitos gera tensão nas fibras, resultando em um material frágil. As características térmicas e morfológicas observadas para as fibras de basalto sugerem sua utilização até temperatura da ordem de no máximo $800^{\circ} \mathrm{C}$, por curto período de tempo [11]. A possibilidade de utilização destas fibras como reforço em compósitos de matriz cerâmica, está sendo explorada em nosso laboratório.

\section{CONCLUSÕES}

Fibras de basalto apresentam estabilidade térmica em relação à perda de massa até $1000{ }^{\circ} \mathrm{C}$. Entretanto, a temperaturas iguais ou superiores a $800{ }^{\circ} \mathrm{C}$ ocorre cristalização acentuada de algumas fases minerais, como a anortita e silicato de magnésio, promovendo alteração da morfologia das fibras, tornando-as frágeis.

\section{AGRADECIMENTOS}

Os autores agradecem os auxílios financeiros das agências FAPESP (2003/009926-1), FAPEMIG (CEX925/04) e CNPq (478563/2004-3 e 300407/2003-3).

\section{REFERÊNCIAS}

[1] C. Potsch, Mineralogia e Geologia, Ed. Livraria Francisco Alves, Rio de Janeiro (1954).

[2] T. Jung, R. V. Subramanian, Scripta Met. Mater. 28 (1993) 527.

[3] J. Militký, V. Kovacic, J. Rubnerová, Eng. Fract. Mech. 69 (2002) 1025.

[4] J. Bernard, G. Berthier, H. Furtak, M. Opozda, Processo de obtenção de fibras minerais, dispositivo de fibração e fibras de basalto, PI9104665, Saint-Gobain, (1990).

[5] O. O. Medvedyev, Y. L. Tsubulya, Proc. 49 ${ }^{\text {th }}$ Int. Symp. Exhib. Mater. Proc. Techn., SAMPE, Long Beach, CA, USA (2004). 
[6] N. N. Morozov, V. S. Bakunov, E. N. Morozov, L. G. Aslanova, P. A. Granovskii, V. V. Prokshin, A. A., Zemlyanitsyn, Glass and Ceramics 58 (2001) 100.

[7] L. V. Toropina, G. G. Vasyuk, O. F. Fedorova, V. L. Kornyushina, V. M. Dyaglev, Y. M. Rassadin, Fiber Chem. 26 (1994) 414.

[8] L. V. Toropina, G. G. Vasyuk, V. L. Kornyushina, V. M. Dyaglev, Y. M. Rassadin, M. A. Makarushina, Fiber Chem. 27 (1995) 67.

[9] J. S. Szabó, T. Czigány, Polymer Testing 22 (2003) 711. [10] Q. Liu, M. T. Shaw, R. S. Parnas, A. McDonnell, Polymer Composites 27, 1 (2006) 41.
[11] V. P. Sergeev, Y. N. Chuvashov, O. V. Galushchak, I. G. Pervak, N. S. Fatikova, Powder Met. Metal Ceram. 33 (1994) 555.

[12] T. Czigány, K. Poloskei, J. Karger-Kocsis, J. Mater. Sci. 40 (2005) 5609.

[13] M. A. Schiavon, L. C. de Souza, L. C. Pardini, I. V. P. Yoshida, Anais XVI Cong. Bras. Enge. Ci. Mater., Porto Alegre, RS (2004) 857.

[14] J. Sim, C. Park, D. Young Moon, Composites: Part B 36 (2005) 504.

[15] P. I. Bashtannik, A. I. Kabak, Y. Y. Yakovchuk, Mechanics of Composite Mater. 39, 1 ( 2003) 85.

(Rec. 28/10/2006, Ac. 16/03/2007) 